\title{
Mechanisms of Antimicrobial Resistance in Pseudomonas aeruginosa and a Multi-Pronged Approach to Combat its Infection in Veterinary Science and Public Health: A Review
}

\author{
Bahar-e-Mustafa ${ }^{1}$, Muhammad Adnan Ashraf ${ }^{2 *}$, Muhammad Khalid Bashir ${ }^{3,4}$ and Hira Batool ${ }^{5}$ \\ ${ }^{1}$ University of Agriculture, Faisalabad. Sub-Campus Toba Tek Singh, Pakistan \\ ${ }^{2}$ Institute of Microbiology, University of Veterinary and Animal Sciences, Lahore, Pakistan \\ ${ }^{3}$ Animal Breeding and genetics, University of Agriculture, Faisalabad \\ ${ }^{4}$ Directorate of Graduate Studies, University of Agriculture, Faisalabad \\ ${ }^{5}$ Chughtai Lab, 10 Jail Road, Main Gulberg, Lahore Pakistan \\ *For correspondence: adnan.ashraf@uvas.edu.pk \\ Received 13 January 2021; Accepted 27 March 2021; Published 10 June 2021
}

\begin{abstract}
Pseudomonas aeruginosa is one of the most important nosocomial pathogens associated with a variety of medical and veterinary infections and therefore, it presents a major public health threat. Different classes of antibiotics are being used to treat its infections which are increasing selective pressure to multi-drug resistance development. Resistance to antibiotics in $P$. aeruginosa is due to many of the common and unique mechanisms which include: reducing membrane permeability, modification or inactivation of antibiotics, alteration of enzymes, modification of target sites and over-expression of efflux systems. Over or under expression of the genes of porin channels and components of efflux systems play a major role in the resistance mechanisms of $P$. aeruginosa. To overcome the problem of the emergence of antibiotic resistance, many new strategies are being employed to control infections caused by $P$. aeruginosa. These include the use of herbs/medicinal plants and phage therapy. With the advent of modern technology, the molecular mechanisms of these alternative therapies are being elucidated and may be used in future to treat $P$. aeruginosa infections in humans and veterinary clinics. This review thus highlights the mechanisms of antibiotic resistance of $P$. aeruginosa against the commonly used antimicrobials and also some alternative strategies to control $P$. aeruginosa infection. (C) 2021 Friends Science Publishers
\end{abstract}

Keywords: Pseudomonas; Antibiotic resistance; Efflux pump; Disinfectant; Porin

\section{Introduction}

Antimicrobial therapy has remained beneficial in treating infectious diseases caused by bacteria. During exposure to antibiotics, pathogens have developed resistance and have become a challenge for healthcare professionals. Among Gram-negative bacteria, Escherichia coli, Acinetobacter baumanni and Klebsiella pneumoniae have become resistant to many antibiotics but the most important pathogen which has shown resistance to all classes of antibiotics is Pseudomonas aeruginosa ( $P$. aeruginosa) (Breidenstein et al. 2011). The organism is a gram-negative; motile bacterium that lacks fermentative properties (which differentiates it from Enterobacteriaceae). It can grow in harsh conditions with low levels of nutrients, bearing the temperature range from $4^{\circ} \mathrm{C}$ to $44^{\circ} \mathrm{C}$. It is an opportunistic microorganism, which is associated with a variety of infections including cystitis, pneumonitis, gastritis, otitis and keratitis in immunocompromised individuals. It is not present in normal human microflora, but it is one of the most notorious organisms responsible for nosocomial infections. It colonizes moist places in hospitals including benchtop surfaces, surgical instruments, urinary catheters and intravenous catheters. P. aeruginosa can survive in disinfectants, making it unique as compared to other pathogens (Chakraborty et al. 2016). Chances of its infection increase with the increase in time of hospitalization (Ferstl et al. 2016).

A variety of factors play roles in the pathogenesis of $P$. aeruginosa including virulence factors. Factors associated with its surface are lipopolysaccharide, rhamnolipids, flagella, mucus and fimbriae, while enzymatic virulence factors include alkaline protease, elastase, hemolysin, neuraminidase, gelatinase, and phospholipases (van 't Wout et al. 2015). These virulence factors help in evading the human immune system and establishing the infection (Khalil et al. 2015). Mechanisms of its resistance are intrinsic as well as extrinsic, depending upon the nature of

To cite this paper: Bahar-e-Mustafa, MA Ashraf, MK Bashir, H Batoool (2021). Mechanisms of antimicrobial resistance in Pseudomonas aeruginosa and a multi-pronged approach to combat its infection in veterinary science and public health: a review. Intl J Agric Biol 26:1-8 
genes involved. Intrinsic mechanisms involve genes from its chromosome while acquired resistance can be obtained from plasmids or bacteriophages, by horizontal gene transfer. Infections of $P$. aeruginosa are difficult to control due to its wide variety of virulence factors and resistance mechanisms. In this review, we highlight the resistance mechanisms against different antibiotics. Understanding of these mechanisms will help in the judicious use of antibiotics and the study of new ways to control this pathogen.

\section{Mechanisms of antibiotic resistance}

All antimicrobials must penetrate the cell wall of a bacterium to be effective. Pseudomonas aeruginosa is resistant to a variety of antibiotics, primarily because it offers limited entry to different antimicrobials. It has a polysaccharide barrier (alginate) around it, which is anionic and can effectively bind with the cationic antimicrobials $e . g$. aminoglycosides and restricts their entry into the cell (Germoni et al. 2016). Moreover, the outer membrane of $P$. aeruginosa also acts as a major barrier in the passage of different molecules which are larger and hydrophilic in nature. Hydrophilic antimicrobials of small molecular size can easily cross the outer membrane of the bacteria by passage through porins (Song et al. 2015).

\section{Decreasing permeability}

Outer membranes of Gram-negative bacteria contain heavy outer membrane proteins in them. These proteins are porin channel i.e., oprJ, oprN, oprM, oprN which help in the transport of molecules but resist the antimicrobials physically or in combination with active efflux pumps. Pseudomonas aeruginosa provides less membrane permeability to antimicrobials as compared to the members of Enterobacteriaceae due to the high ratio of porins. Porin proteins and efflux pumps work side by side to increase their resistance. oprF is a major type of porin produced by all strains of the $P$. aeruginosa. OprD is another important porin involved principally in the uptake of lysine (and other positively charged amino acids). If this porin is lost, it results in the resistance to the meropenem (Fluit et al. 2019) and carbapenem (Richardot et al. 2015). Another porin is OprH which prevents the binding of antimicrobials to the lipopolysaccharides (LPS) of bacteria thus preventing the uptake of those compounds (Qadi et al. 2016).

\section{Efflux system (multidrug)}

Different families of efflux system are the protein pumps involved in resistance for the transport of substances across the bacterial membrane and cell wall. One of the families is resistance-nodulation-division (RND) which characterizes both impermeability resistance and adaptive resistance to multiple antibiotics in $P$. aeruginosa. It is primarily composed of three major components that include 1) an inner membrane pump (the RND component), 2) An outer membrane channel-forming porin and 3) a periplasmic linker protein which joins the two components together (Fig. 1A, B, C, D). While writing the name of the efflux pump, periplasmic linker and the inner membrane pump come first followed by the type of porin (Daury et al. 2016). Different types of efflux systems which are reported for the $P$. aeruginosa are mexAB-oprM (mexA = periplasmic linker, $\operatorname{mexB}=$ membrane pump, oprM = porin $)$, mexCDoprJ, mexEF-oprN, mexXY-oprM and mexXY-oprN. The most commonly present efflux pump is the mexAB-oprM while mexXY-oprN is less commonly found. The activity of mexXY-oprN is dependent on the presence or absence of porin oprD, as its range of resistance is increased with the absence of oprD porin (Kao et al. 2016). Common types of efflux pumps are mentioned in Table 1.

MexAB-OprM efflux system participates in both intrinsic as well as acquired resistance in $P$. aeruginosa, but MexEF-OprN and MexCD-OprJ contribute only to the acquired resistance. Role of these efflux pumps was confirmed by the knock-out gene mutations. At the start, oprK was considered to be the porin channel of MexAB efflux pump and was referred as MexAB-oprK pump but later on, oprM was found to be the porin of MexAB efflux pump and named as MexAB-oprM efflux pump (Baranova 2016). Moreover, the efflux system also plays an important role in inducing resistance against meropenem (Rostami et al. 2018).

\section{Modification and inactivation of antibiotics}

The AmpC gene is possessed by all Pseudomonas strains. This gene primarily produces resistance to beta-lactam antibiotics. AmpR is a regulatory gene which is responsible for the overproduction of beta-lactamase by $P$. aeruginosa strains. Beta-lactamase is present in the periplasmic space of $P$. aeruginosa. Beta-lactamases, as well as integron and plasmid-encoded extended-spectrum beta-lactamases (ESBLs), are responsible for contributing resistance against cephalosporin and penicillin. Beta-lactamase inhibitors interfere with the enzyme by plasmid but not by AmpC gene (Buberg et al. 2020).

In the case of $P$. aeruginosa, this mechanism has been observed against quinolones and penicillins. Quinolones bind to DNA gyrase enzyme. A gyrA mutation results in the change of the DNA gyrase enzyme, thus making quinolones ineffective (Park et al. 2020). Pseudomonas strains associated with cystic fibrosis exhibit a frequent change in the penicillin-binding proteins (PBP's) resulting in the resistance against the penicillin group. mRNA expression levels of oprM and ampC genes led to 21.7 and $25 \%$ resistance respectively. Point mutation of AmpR at Asp135-Asn and Als194-Ser deregulated the ampC induction and led to $21.7 \%$ resistance in Pseudomonas isolates (Du et al. 2010). 
Table 1: Common types of multi-drug resistance efflux pumps with their resistant and susceptible antibiotics

\begin{tabular}{|c|c|c|c|}
\hline $\begin{array}{l}\text { Periplasmic } \\
\text { Linker }\end{array}$ & $\begin{array}{l}\text { Inner membrane Porin } \\
\text { Pump }\end{array}$ & Resistance to Antimicrobials & Antimicrobials not affected \\
\hline MexA & MexB OprM & $\begin{array}{l}\text { Quinolone, Macrolide, Tetracycline, Chloramphenicol, anti-pseudomonal Penicillins, } \\
\text { anti-pseudomonal Cephalosporins, Disinfectants }\end{array}$ & Aminoglycoside, Imipenem \\
\hline $\mathrm{MexC}$ & MexD & Quinolone, Macrolide, Tetracycline, Chloramphenicol, & Carbenicillin, Sulbenicillin, Ceftazidime \\
\hline MexE & MexF & Fluoroquinolone, Chloramphenicol, Trimethoprim, Carbapenems & $\begin{array}{l}\text { Ticarcillin, Cefepime, Aztreonam, } \\
\text { Aminoglycoside }\end{array}$ \\
\hline MexX & MexY & Quinolone, Macrolide, Tetracycline, Chloramphenicol, $\beta$-lactams, Aminoglycosides & $\begin{array}{l}\text { Carbenicillin, Sulbenicillin, Cefsulodin, } \\
\text { Ceftazidime }\end{array}$ \\
\hline
\end{tabular}

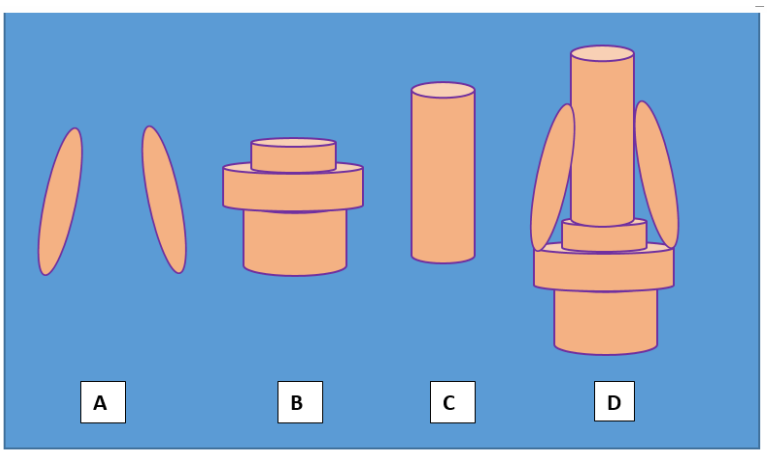

Fig. 1: A; Periplasmic linker protein, B; Inner membrane pump, C; Channel porin, $\mathbf{D}$; Complete Efflux pump

\section{Induction of biofilm}

Pseudomonas aeruginosa colonization appears as the aggregate of the cells, surrounded by the protective coating of polysaccharide, alginate, proteins and extracellular DNA. These biofilms are becoming a major source of resistance against antibiotics and disinfectants (Hameed et al. 2017). Pseudomonas aeruginosa effectively forms biofilms when exposed to the sub-optimal concentrations of the different antibiotics like tobramycin, tetracycline and ciprofloxacin. Tobramycin is also responsible for the induction of swimming and swarming in P. aeruginosa (Linares et al. 2006). Biofilm resists by interacting with different mechanisms simultaneously. Biofilm matrix allows limited penetration of antibiotics towards bacterial cells. Bacterial cells produce a limited number of inactive cells in the biofilm matrix that do not grow or die even in the presence of antibiotics, called persister cells. These cells have the special ability to tolerate multiple antibiotics after their diffusion through the matrix (Dawson et al. 2011). Extracellular DNA having negative charge binds with the cationic antimicrobial peptides including polymyxin to restrict their entry into the cell. Moreover, exposure of pseudomonal biofilms to the imipenem resulted in enhanced expression of genes responsible for the biosynthesis of alginate, which results in the production of stronger biofilm formation (Olivares et al. 2020).

\section{Resistance against beta-lactam ring containing antibiotics}

$P$. aeruginosa is resistant against many compounds which may be structurally related or unrelated. Intrinsically, it is resistant to many antimicrobial compounds due to $\beta$ lactamases against penicillin $\mathrm{G}$, aminopenicillins and cephalosporins. Beta-lactam antibiotics lead to induction of the AmpC gene which is chromosomally encoded but horizontal gene transfer increases resistance as well. The incidence of ES $\beta \mathrm{L}$ resistant strains between $P$. aeruginosa isolates was found 26.86 and $25.14 \%$ in nosocomial and community sources, respectively (Sahu et al. 2012). Moreover, 25.3 and $28.7 \%$ P. aeruginosa isolates were resistant to meropenem and imipenem (Hu et al. 2017). The resistance is due to outer membrane permeability and efflux pumps (transporters) that actively push beta-lactam antibiotics out of the cell and also got the ability to contain some of the enzymes which can inactivate the antimicrobial drugs e.g., penicillinases and cephalosporinases ( $\mathrm{Xu}$ et al. 2020). Carbapenems are considered as last resort antibiotics for the treatment of $P$. aeruginosa along with colistin (Manohar et al. 2018).

Induction of AmpC gene and the extent of hydrolysis of antibiotics decide the fate of beta-lactams against $P$. aeruginosa. Amoxicillin, $1^{\text {st }}$ and $2^{\text {nd }}$ generation cephalosporins are relatively ineffective against $P$. aeruginosa infections due to their easy hydrolysis and strong induction of AmpC. An important group of betalactam antibiotics that are used to treat the infection of $P$. aeruginosa include ureidopenicillins (azlocillin), carboxypenicillins (ticarcillin and carbenicillin), $3^{\text {rd }} \& 4^{\text {th }}$ generation cephalosporins (ceftazidime and cefoperazone), aztreonams and carbapenems (meropenem and imipenem) (Bagge et al. 2002). This is leading to the appearance of new $\beta$-lactamases by natural evolution.

\section{Aminoglycosides}

Aminoglycosides are bactericidal and they do this by binding to the $30 \mathrm{~S}$ subunit of bacterial ribosomes, misreading of the codons and thus causing the death of microorganisms (Dunkle et al. 2014). Pseudomonas aeruginosa is naturally resistant to some of the aminoglycosides like kanamycin because it can easily phosphorylate it (Kondo and Hotta 1999). The uptake of the aminoglycosides is a complex process which involves binding with LPS and permeability offered by the outer membrane of Gram-negative bacteria, then crossing of substances across the plasma membrane due to action potential and ultimately binding with ribosomes and disrupting the polypeptide synthesis in bacteria 
(Krahn et al. 2012). Pseudomonas aeruginosa has developed resistance which causes methylation of nucleotides due to horizontal gene transfer.

Strains of $P$. aeruginosa isolated from the clinical and laboratory studies have shown both intrinsic and adaptive type of resistance against aminoglycosides. It is well documented that aminoglycosides can also be antagonized by certain ions of divalent nature i.e., $\mathrm{Ca}$ and $\mathrm{Mg}$ particularly in the case of $P$. aeruginosa (Morita et al. 2012). A recent analysis at the transcriptomic level has shown that aminoglycosides can affect the variety of the genes of intrinsic and adaptive nature. Pseudomonas aeruginosa, when exposed to the tobramycin for quite a prolonged interval of time at a dose less than MIC can significantly enhance the expression of MexXY (efflux pump) genes. Heat shock genes (i.e., groES, asrA, htpG and ibpA) are also overexpressed when $P$. aeruginosa is exposed to tobramycin at a sub-lethal concentration; among them, asrA is particularly important regarding resistance to aminoglycosides (Basta et al. 2020). Expression of chromosomal genes mexZ, rplY, PA5471, nuoG \& galU, and MexXY-OprM efflux system increase aminoglycoside resistance in $P$. aeruginosa (Islam et al. 2009).

\section{Resistance against tetracycline}

Tetracycline group is primarily bacteriostatic in action and includes a variety of antimicrobial compounds. An energydriven process is required to enter the tetracyclines inside the cell, where they bind to the $30 \mathrm{~S}$ ribosomal subunit and interfere with the binding of aminoacyl-tRNA to the A site (acceptor) in the ribosomal-RNA complex. Intrinsically tetracyclines are ineffective against Pseudomonal infections because of the presence of the MexAB/mexXY multidrug efflux type of pumps (Konai and Haldar 2020).

\section{Resistance against macrolides}

Macrolides are a group of antimicrobials that also tend to interfere with the synthesis of proteins in microorganisms and they do this by attaching to the 50S subunit of ribosomes. These are commonly used to treat the pulmonary infections associated with $P$. aeruginosa (Laserna et al. 2014; Solleti et al. 2015). They also tend to induce type III secretion system of the bacteria. Low concentration (2 $\mu \mathrm{g} / \mathrm{mL}$ ) of macrolides enhances the production of some mutant strains e.g. $n f x B$ mutants, which then effectively produce the efflux pumps i.e., MexCD-OprJ. Chromosomally encoded expression of MexCD-OprJ and MexXY/MexAB-OprM efflux pumps interferes with the natural resistance of $P$. aeruginosa against the macrolide (Mulet et al. 2011).

\section{Resistance against chloramphenicol}

Chloramphenicol is primarily bacteriostatic that interferes with the multiplication of the microorganisms by binding to the 50S subunit of the bacterial ribosomes and they inhibit the peptidaltransferase enzyme. Pseudomonas is resistant against chloramphenicol intrinsically, mainly due to the presence of an efflux system of MexAB-oprM type (Fernández et al. 2012). Moreover, the sub-optimal doses of the drug lead to the induction of MexXY efflux system.

\section{Resistance against fluoroquinolones}

MexXY-OprM and MexAB-OprM contribute to quinolone resistance in wild-type Pseudomonal spp. (Morita et al. 2001). Efflux pumps have been found that are responsible for the resistance development in case of Pseudomonas i.e., MexVW-OprM and MexHI-OpmD. MexEF-OprN and MexCD-OprJ efflux pumps are linked to quinolone resistance while MexCD-OprJ is related to multidrug resistance (Terzi et al. 2014).

\section{Resistance against other biocides}

Triclosan, chlorhexidine and benzalkonium can become contaminated with the Pseudomonas, therefore, making the disinfectant less efficient (Shepherd et al. 2018). Resistance against triclosan is primarily due to an efflux pump i.e., MexAB-OprM (Mima et al. 2007; Zhu et al. 2010). The suboptimal concentration of the chlorhexidine and benzalkonium lead to the resistance against Pseudomonas primarily by MexCD-OprJ efflux pump. Pseudomonas aeruginosa strains showing resistance against the benzalkonium also tend to become resistant against the quinolones due to mutations in the gyrA, mexCD-oprJ and mexAB-oprM (Mc Cay et al. 2010). Resistance mechanisms of $P$. aeruginosa have been summarized in Table 2 .

\section{Implications of anti-microbial resistance in veterinary and public health and employing alternative intervention strategies}

In humans, $P$. aeruginosa is known to cause a myriad of clinical diseases such as sepsis, pneumonia, skin infections and cystic fibrosis (Jeong et al. 2014). So, these infections contribute significantly towards the higher morbidity, mortality and treatment cost. According to China antimicrobial surveillance network (CHINET), $P$. aeruginosa is one of the four Gram-negative bacteria to be isolated from each clinical specimen (Hu et al. 2017). In animals, $P$. aeruginosa is known to cause many diseases, including, chronic pyoderma, Urinary tract infections (UTIs), wound infections, otitis externa, bovine mastitis and feline septicemia (Ahmad 2001; Mekić et al. 2011; Maniam et al. 2019). $P$. aeruginosa isolated from the cats and dogs in Japan have shown 4.1, 12.3, 17.8, 20.5, 31.5 and 34.2\% resistance against gentamicin, aztreonam, cefotaxime, ciprofloxacin, enrofloxacin and orbifloxacin. Ear isolates of $P$. aeruginosa have shown resistance to orbifloxacin, enrofloxacin and ciprofloxacin. Moreover, bacteria from 
Table 2: Resistance mechanisms of $P$. aeruginosa against commonly used antimicrobials

\begin{tabular}{lll}
\hline Antimicrobial & Mechanism & Reference \\
\hline$\beta$ - lactams & Induction of AmpC $\beta$ - lactamase & (Sahu et al. 2012) \\
Aminoglycosides & Expression of Heat shock genes; Alteration in MexXY-OprM & (Islam et al. 2009; Basta et al. 2020) \\
& Biofilm development & (Linares et al. 2006) \\
& Induction of swimming and swarming & (Linares et al. 2006) \\
Carbapenems & Development of thicker biofilm & (Olivares et al. 2020) \\
Chloramphenicol & Induction of Mex EF-OprN complex & (Fernández et al. 2012) \\
quinolones & Over-expressed MexEF-OprN and MexCD-OprJ & (Terzi et al. 2014) \\
Tetracycline & Over-expressed mexA-mexB-oprM & (Konai and Haldar 2020) \\
MDR & MexCD-OprJand MexAB-OprM & (Terzi et al. 2014) \\
\hline
\end{tabular}

urine were more resistant to many antimicrobial drugs than skin isolates (Harada et al. 2012). Similar findings have been demonstrated in a study that showed that $P$. aeruginosa isolated from canine otitis externa to be 47, 67 and $75 \%$ resistant against enrofloxacin, marbofloxacin and ciprofloxacin (Wildermuth et al. 2007). Similar trends of antimicrobial resistance have been shown in the $P$. aeruginosa isolates from mouth, skin, urogenital tract and ears (Werckenthin et al. 2007). In the recent past, it has been demonstrated that $P$. aeruginosa can cross different species barriers thereby indicating its potential as a zoonotic pathogen (Fernandes et al. 2018).

So, because of the presence of multidrug resistance in $P$. aeruginosa, there is a need for appropriate drug therapy to overcome this issue, because inappropriate antimicrobial therapy at initial disease stages can lead to enhanced morbidity and mortality. So, as done in the past, combinations of antibiotics (although controversial) may be employed to deal with the problem of multidrug resistance (Guan et al. 2016). Although colistin and amikacin exhibit anti-pseudomonal activity, but their toxicity deters their frequent use in clinics. So, in this aspect, new antibiotics that are known to possess activity against the multi-drug resistance (MDR) P. aeruginosa, they may be deployed prudently in clinics to curb the infection by MDR $P$. aeruginosa. Tazobactam/ Ceftolozane is a very effective antibacterial drug combination that is recommended to treat the intra-abdominal infections as well as UTIs. In vitro studies have demonstrated that the development of antimicrobial resistance against this combination is slower as compared to the other antibiotics (Tato et al. 2015). Moreover, a new combination of avibactam (non betalactam) with the $3^{\text {rd }}$ generation cephalosporin (Ceftazidime) has also been approved for the treatment of UTIs and intraabdominal infections (along with metronidazole) caused by P. aeruginosa (Sader et al. 2017; Xipell et al. 2017). Another study has also demonstrated that after colistin, the combination of Tazobactam/Ceftolozane and avibactam/Ceftazidime was found to be most effective against $P$. aeruginosa infections where 97.5 and $96.9 \%$ of $P$. aeruginosa population was found to be susceptible to both combinations, respectively (Sader et al. 2018). So, there is still much room to discover some novel antimicrobial combinations which may prove good antimicrobial activity both in vitro and under clinics against $P$. aeruginosa.
Alternatively, another approach to combat infections caused by $P$. aeruginosa may be by using various plant/herb based compounds, and this approach is much safer than using antimicrobials. Medicinal plants or herbs have a variety of compounds such as long-chained unsaturated aldehydes, peptides, essential oils, and phenolic compounds, which are known to possess the antimicrobial activity (Hameed and Ahmed 2014; Astal et al. 2005). It has been shown that ginseng supplementation results in the reduced $P$. aeruginosa and mast cells number in the lungs of rats, thereby significantly reducing the lung pathology (Nguyen and Nguyen 2019). Leave extract of Azadirachta indica (neem) are also found to inhibit the biofilms formation in $P$. aeruginosa (Harjai et al. 2013). One more study demonstrated that flavonoids extracted from Moringa oleifera possess the anti-biofilm activities of $P$. aeruginosa (Onsare and Arora 2015). Another study has found that Zingiber officinale (ginger), Glycyrrhiza glabra (liquorice) and Mentha piperita (mint), possess antipseudomonal activity against many MDR strains (Chakotiya et al. 2016). Although the exact mechanism by which these herbs/nutraceuticals impart antimicrobial effects largely remains to be elucidated, however, their phytochemicals play important role in the molecular mechanisms involved in their antimicrobial effects. In vivo mice model studies have shown that oral supplementation of garlic extract is useful in preventing the infections caused by $P$. aeruginosa. After oral administration, garlic was found to be effective in reducing the renal bacterial count. In vitro studies demonstrated the reduction in the signals responsible for quorum sensing and thereby reducing the release of various virulence factors (Harjai et al. 2010). A more recent study also demonstrated that purified flavonoids fraction derived from Cassia alata L. (Ca. alata), was found to be associated with the reduced release of virulence factors, reduction in quorum sensing signals and reduced biofilm formation by $P$. aeruginosa (Rekha et al. 2017). The latest study has demonstrated that quercetin (natural flavonoid compound) has been found to specifically inhibit the quorum sensing 
and thus biofilm formation and virulence factors production by $P$. aeruginosa (Ouyang et al. 2016).

Recently phage therapy presented an alternative solution to multidrug-resistant bacteria and it offers several advantages over antibiotics. A single dose of phage can eradicate potentially infectious bacteria. However, till now, phages are reported to be genera and species (even strain) specific (Nguyen et al. 2012). Siphoviridae phages are known to be important in controlling $P$. aeruginosa infections(Yamaguchi et al. 2014). In a recent study, JHP phage has shown its antibacterial activity against a myriad of $P$. aeruginosa strains (Khawaja et al. 2016).

\section{Conclusion}

Resistance mechanisms are constantly evolving by natural evolution or horizontal gene transfer. There is a grave challenge to tackle infections these days as the antibiotic resistance keeps on increasing from multidrug to pan-drug resistance. These resistant strains can be treated by aggressive approach, avoiding the suboptimal doses. Infections should be treated with broad-spectrum antibiotics followed by narrowing down to the specific antibiotic till the sensitivity result comes. Antibiotic therapy can be used with supporting agents as beta-lactamase inhibitors e.g. Clavulanate or biofilm dissolving substances $e . g$., alginate lyases. There is also need to focus on the post-antibiotic era; screening drugs for efflux pump inhibitors, searching new types of beta-lactamase inhibitors, exploring cationic membrane permeabilizers, manipulating medicinal plants/herbs, enhancing quorum quenching to inhibit communication and biofilm formation and phage therapy to treat infections. Emphasis should also be done to improve the specific or general immune response of patients infected by $P$ aeruginos $a$ along with other non-fermentative bacterial infections.

\section{Author Contributions}

BEM and MAA conceived the idea, BEM acquired the data, BEM and MAA wrote the manuscript with the inputs of MKB and HB. MKB and HB worked on drafting and revising of the article critically.

\section{Conflict of Interest}

We declare that we do not have any conflict of interest.

\section{Data Availability}

Not applicable.

\section{Ethics Approval}

Not applicable.

\section{Funding Source}

The study did not receive any funding.

\section{References}

Ahmad R (2001). Studies on mastitis among dairy buffaloes. Pak Vet $J$ 21:220-221

Astal EZ, A Aera, K Aam (2005). Antimicrobial activity of some medicinal plant extracts in Palestine. Pak J Med Sci 21:187-193

Bagge N, O Ciofu, M Hentzer, JIA Campbell, M Givskov, N Hoiby (2002). Constitutive high expression of chromosomal lactamase in Pseudomonas aeruginosa caused by a new insertion sequence (IS1669) located in ampD. Antimicrob Agents Chemother 46:3406-3411

Baranova N (2016). Involvement of antimicrobial drug efflux systems in bacterial fitness and virulence. In: Efflux-Mediated Antimicrobial Resistance in Bacteria, pp:701-727. Adis, Cham, Switzerland

Basta DW, D Angeles-Albores, MA Spero, JA Ciemniecki, DK Newman (2020). Heat-shock proteases promote survival of Pseudomonas aeruginosa during growth arrest. Proc Natl Acad Sci USA 117:4358-4367

Breidenstein EBM, C de la Fuente-Núñez, REW Hancock (2011). Pseudomonas aeruginosa: All roads lead to resistance. Trends Microbiol 19:419-426

Buberg ML, IL Witsø, TM L'Abée-Lund, Y Wasteson (2020). Zinc and copper reduce conjugative transfer of resistance plasmids from extended-spectrum beta-lactamase-producing Escherichia coli. Microb Drug Resist 26:842-849

Chakotiya AS, R Chawla, P Thakur, A Tanwar, A Narula, SS Grover, R Goel, R Arora, RK Sharma (2016). In vitro bactericidal activity of promising nutraceuticals for targeting multidrug resistant Pseudomonas aeruginosa. Nutrition 32:890-897

Chakraborty B, S Chatterjee, R Ray, N Pal, S Patra, P Maiti (2016). Rethink on recommended concentrations of disinfectants in the light of biofilm, based on in vitro study. Intl J Heal Allied Sci 5:154-158

Daury L, F Orange, JC Taveau, A Verchère, L Monlezun, C Gounou, RKR Marreddy, M Picard, I Broutin, KM Pos, O Lambert (2016). Tripartite assembly of RND multidrug efflux pumps. Nat Commun 7; Article 10731

Dawson CC, C Intapa, MA Jabra-Rizk (2011). Persisters: Survival at the cellular level. PLoS Pathog 7; Article e1002121

Du SJ, HC Kuo, CH Cheng, ACY Fei, HW Wei, SK Chang (2010). Molecular mechanisms of ceftazidime resistance in Pseudomonas aeruginosa isolates from canine and human infections. Vet Med 55:172-182

Dunkle JA, K Vinal, PM Desai, N Zelinskaya, M Savic, DM West, GL Conn, CM Dunham (2014). Molecular recognition and modification of the $30 \mathrm{~S}$ ribosome by the aminoglycoside-resistance methyltransferase NpmA. Proc Natl Acad Sci 111:6275-6280

Fernandes MR, FP Sellera, Q Moura, MPN Carvalho, PN Rosato, L Cerdeira, N Lincopan (2018). Zooanthroponotic transmission of drug-resistant Pseudomonas aeruginosa, Brazil. Emerg Infect Dis 24:1160-1162

Fernández M, S Conde, JDL Torre, C Molina-Santiago, JL Ramos, E Duque (2012). Mechanisms of resistance to chloramphenicol in Pseudomonas putida KT2440. Antimicrob Agents Chemother 56:1001-1009

Ferstl P, N Filmann, C Brandt, S Zeuzem, T Wichelhaus, M Hogardt, V Kempf, O Waidmann, C Reinheimer (2016). Colonization and infection with carbapenem-resistant non-fermenting gram-negative bacteria are associated with rapid deterioration and mortality in patients with decompensated liver disease. Zeitsch Gastroenterol 54:KV268

Fluit AC, RJ Rentenaar, MB Ekkelenkamp, TT Severs, AMC MavinkurveGroothuis, MRC Rogers, MCA Bruin, TFW Wolfs (2019). Fatal carbapenem resistance development in Pseudomonas aeruginosa under meropenem monotherapy, caused by mutations in the oprD outer membrane porin. Pediatr Infect Dis J 38:398-399 
Germoni LAP, PJ Bremer, IL Lamont (2016). The effect of alginate lyase on the gentamicin resistance of Pseudomonas aeruginosa in mucoid biofilms. J Appl Microbiol 121:126-135

Guan X, L He, B Hu, J Hu, X Huang, G Lai, Y Li, Y Liu, Y Ni, H Qiu, Z Shao, Y Shi, M Wang, R Wang, D Wu, C Xie, Y Xu, C Zhuo (2016). Laboratory diagnosis, clinical management and infection control of the infections caused by extensively drug-resistant Gram-negative Bacilli: A Chinese consensus statement. Clin Microbiol Infect 22:15-25

Hameed H, SI Ahmed (2014). The role of chemical and herbal antipathogenic compounds in the prevention of quorum sensingdependent pathogenicity of Pseudomonas aeruginosa-A Review. Pak Vet J 34:426-431

Hameed H, I Hussain, MS Mahmood, F Deeba, K Riaz (2017). Higher order occurrence of virulent isolates of Pseudomonas aeruginosa in hospital environments initiate one health concerns irrespective of the biological association. Pak Vet J 37:7-12

Harada K, S Arima, A Niina, Y Kataoka, T Takahashi (2012). Characterization of Pseudomonas aeruginosa isolates from dogs and cats in Japan: Current status of antimicrobial resistance and prevailing resistance mechanisms. Microbiol Immunol 56:123-127

Harjai K, A Bala, RK Gupta, R Sharma (2013). Leaf extract of Azadirachta indica (neem): A potential antibiofilm agent for Pseudomonas aeruginosa. Pathog Dis 69:62-65

Harjai K, R Kumar, S Singh (2010). Garlic blocks quorum sensing and attenuates the virulence of Pseudomonas aeruginosa. FEMS Immunol Med Microbiol 58:161-168

HuF, Y Guo, D Zhu, F Wang, X Jiang, Y Xu, X Zhang, Z Zhang, P Ji, Y Xie, M Kang, C Wang, A Wang, Y Xu, J Shen, Z Sun, Z Chen, J Meng (2017). CHINET surveillance of bacterial resistance across China: Report of the results in 2016. Chin J Infect Chemother 17:481-491

Islam S, H Oh, S Jalal, F Karpati, O Ciofu, N Høiby, B Wretlind (2009). Chromosomal mechanisms of aminoglycoside resistance in Pseudomonas aeruginosa isolates from cystic fibrosis patients. Clin Microbiol Infect 15:60-66

Jeong J, Y Cho, H Lee, M Kim, N Kim, H Song, K Lee (2014). Multifocal discospondylitis in a male dog with prostatic abscess and cystitis. Pak Vet J 34:566-568

Kao CY, SS Chen, KH Hung, HM Wu, PR Hsueh, JJ Yan, JJ Wu (2016). Overproduction of active efflux pump and variations of OprD dominate in imipenem-resistant Pseudomonas aeruginosa isolated from patients with bloodstream infections in Taiwan. BMC Microbiol 16; Article 107

Khalil MAEF, FI Sonbol, AFB Mohamed, SS Ali (2015). Comparative study of virulence factors among ES $\beta$ L-producing and nonproducing Pseudomonas aeruginosa clinical isolates. Turk J Med Sci 45:60-69

Khawaja KA, M Rauf, Z Abbas, S ur Rehman (2016). A virulent phage JHP against $P$ seudomonas aeruginosa showed infectivity against multiple genera. J Basic Microbiol 56:1090-1097

Konai MM, J Haldar (2020). Lysine-based small molecule sensitizes rifampicin and tetracycline against multidrug-resistant Acinetobacter baumannii and Pseudomonas aeruginosa. ACS Infect Dis 6:91-99

Kondo S, K Hotta (1999). Semisynthetic aminoglycoside antibiotics: Development and enzymatic modifications. J Infect Chemother 5:1-9

Krahn T, C Gilmour, J Tilak, S Fraud, N Kerr, CH-F Lau, K Poole (2012). Determinants of intrinsic aminoglycoside resistance in Pseudomonas aeruginosa. Antimicrob Agents Chemother 56:5591-5602

Laserna E, O Sibila, JF Fernandez, DJ Maselli, EM Mortensen, A Anzueto, G Waterer, MI Restrepo (2014). Impact of macrolide therapy in patients hospitalized with Pseudomonas aeruginosa communityacquired pneumonia. Chest 145:1114-1120

Linares JF, I Gustafsson, F Baquero, JL Martinez, GIBFMJ Linares JF (2006). Antibiotics as intermicrobial signaling agents instead of weapons. Proc Natl Acad Sci USA 103:19484-19489

Maniam R, A Salleh, ZS Mohd, J Faez, F Abdullah, Z Zunita (2019). A study of aetiology and risk factors of bacterial septicaemia of cats. Pak Vet J 39:236-240

Manohar P, S Babu, B Bozdogan, N Ramesh (2018). Identification of blaDIM-1 metallo- $\beta$-lactamase gene in Pseudomonas aeruginosa isolated from Tamil Nadu, India. J Glob Antimicrob Resist 13:7-8
Mc Cay PH, AA Ocampo-Sosa, GTA Fleming (2010). Effect of subinhibitory concentrations of benzalkonium chloride on the competitiveness of Pseudomonas aeruginosa grown in continuous culture. Microbiology 156:30-38

Mekić S, K Matanović, B Šeol (2011). Antimicrobial susceptibility of Pseudomonas aeruginosa isolates from dogs with otitis externa. Vet Rec 169:125-129

Mima T, S Joshi, M Gomez-Escalada, HP Schweizer (2007). Identification and characterization of TriABC-OpmH, a triclosan efflux pump of $P$ seudomonas aeruginosa requiring two membrane fusion proteins. $J$ Bacteriol 189:7600-7609

Morita Y, J Tomida, Y Kawamura (2012). MexXY multidrug efflux system of Pseudomonas aeruginosa. Front Microbiol 3; Article 408

Morita Y, N Kimura, T Mima, T Mizushima, T Tsuchiya (2001). Roles of MexXY- and MexAB-multidrug efflux pumps in intrinsic multidrug resistance of Pseudomonas aeruginosa PAO1. J Gen Appl Microbiol 47:27-32

Mulet X, B Moyá, C Juan, MD Macià, JL Pérez, J Blázquez, A Oliver (2011). Antagonistic interactions of Pseudomonas aeruginosa antibiotic resistance mechanisms in planktonic but not biofilm growth. Antimicrob Agents Chemother 55:4560-4568

Nguyen NH, CT Nguyen (2019). Pharmacological effects of ginseng on infectious diseases. Inflammopharmacology 27:871-883

Nguyen HTD, S Yoon, MH Kim, YK Kim, MY Yoon, YH Cho, Y Lim, SH Shin, DE Kim (2012). Characterization of bacteriophage $\phi$ Ptobp6g, a novel phage that lyses Pseudomonas tolaasii causing brown blotch disease in mushrooms. J Microbiol Meth 91:514-519

Olivares E, S Badel-Berchoux, C Provot, G Prévost, T Bernardi, F Jehl (2020). Clinical impact of antibiotics for the treatment of Pseudomonas aeruginosa biofilm infections. Front Microbiol 10; Article 2894

Onsare JG, DS Arora (2015). Antibiofilm potential of flavonoids extracted from Moringa oleifera seed coat against Staphylococcus aureus, Pseudomonas aeruginosa and Candida albicans. J Appl Microbiol 118:313-325

Ouyang J, F Sun, W Feng, Y Sun, X Qiu, L Xiong, Y Liu, Y Chen (2016). Quercetin is an effective inhibitor of quorum sensing, biofilm formation and virulence factors in Pseudomonas aeruginosa. J Appl Microbiol 120:966-974

Park Y, J Oh, S Park, S Sum, W Song, J Chae, H Park (2020). Antimicrobial resistance and novel mutations detected in the gyrA and parC genes of Pseudomonas aeruginosa strains isolated from companion dogs. BMC Vet Res 16; Article 111

Qadi M, C Lopez-Causapé, S Izquierdo-Rabassa, M Mateu Borrás, JB Goldberg, A Oliver, S Albertí (2016). Surfactant protein A recognizes outer membrane protein OprH on Pseudomonas aeruginosa chronic infection isolates. J Infect Dis 214:1449-1455

Rekha PD, HS Vasavi, C Vipin, K Saptami, AB Arun (2017). A medicinal herb Cassia alata attenuates quorum sensing in Chromobacterium violaceum and Pseudomonas aeruginosa. Lett Appl Microbiol 64:231-238

Richardot C, P Plésiat, D Fournier, L Monlezun, I Broutin, C Llanes (2015). Carbapenem resistance in cystic fibrosis strains of Pseudomonas aeruginosa as a result of amino acid substitutions in porin OprD. Intl J Antimicrob Agents 45:529-532

Rostami S, A Farajzadeh Sheikh, S Shoja, A Farahani, MA Tabatabaiefar, A Jolodar, R Sheikhi (2018). Investigating of four main carbapenemresistance mechanisms in high-level carbapenem resistant Pseudomonas aeruginosa isolated from burn patients. J Chin Med Assoc 81:127-132

Sader HS, RK Flamm, CG Carvalhaes, M Castanheira (2018). Antimicrobial susceptibility of Pseudomonas aeruginosa to ceftazidime-avibactam, ceftolozane-tazobactam, piperacillin-tazobactam, and meropenem stratified by U.S. census divisions: Results from the 2017 INFORM program. Antimicrob Agents Chemother 62:1-6

Sader HS, M Castanheira, D Shortridge, RE Mendes, RK Flamm (2017). Antimicrobial activity of ceftazidime-avibactam tested against multidrug-resistant Enterobacteriaceae and Pseudomonas aeruginosa isolates from U.S. Medical Centers, 2013 to 2016. Antimicrob Agents Chemother 61; Article e01045-17 
Sahu MC, D Dubey, S Rath, NK Debata, RN Padhy (2012). Multidrug resistance of Pseudomonas aeruginosa as known from surveillance of nosocomial and community infections in an Indian teaching hospital. J Public Health 20:413-423

Shepherd MJ, G Moore, ME Wand, JM Sutton, LJ Bock (2018). Pseudomonas aeruginosa adapts to octenidine in the laboratory and a simulated clinical setting, leading to increased tolerance to chlorhexidine and other biocides. J Hosp Infect 100:e23-e29

Solleti VS, M Alhariri, M Halwani, A Omri (2015). Antimicrobial properties of liposomal azithromycin for Pseudomonas infections in cystic fibrosis patients. J Antimicrob Chemother 70:784-796

Song J, JCE Odekerken, DWPM Löwik, PM López-Pérez, TJM Welting, F Yang, JA Jansen, SCG Leeuwenburgh (2015). Influence of the molecular weight and charge of antibiotics on their release kinetics from gelatin nanospheres. Macromol Biosci 15:901-911

Tato M, M García-Castillo, AM Bofarull, R Cantón (2015). In vitro activity of ceftolozane/tazobactam against clinical isolates of Pseudomonas aeruginosa and Enterobacteriaceae recovered in Spanish medical centres: Results of the CENIT study. Intl J Antimicrob Agents 46:502-510

Terzi HA, C Kulah, IH Ciftci (2014). The effects of active efflux pumps on antibiotic resistance in Pseudomonas aeruginosa. World J Microbiol Biotechnol 30:2681-2687

van 't Wout EFA, A van Schadewijk, R van Boxtel, LE Dalton, HJ Clarke, J Tommassen, SJ Marciniak, PS Hiemstra (2015). Virulence factors of Pseudomonas aeruginosa induce both the unfolded protein and integrated stress responses in airway epithelial cells. PLOS Pathog 11; Article e1004946
Werckenthin C, E Alesík, M Grobbel, A Lübke-Becker, S Schwarz, L Wieler, J Wallmann (2007). Antimicrobial susceptibility of Pseudomonas aeruginosa from dogs and cats as well as Arcanobacterium pyogenes from cattle and swine as determined in the BfT-GermVet monitoring program 2004-2006. Berl Munch Tierarztl Wochenschr 120:412-422

Wildermuth BE, CE Griffin, WS Rosenkrantz, MJ Boord (2007). Susceptibility of Pseudomonas isolates from the ears and skin of dogs to enrofloxacin, marbofloxacin, and ciprofloxacin. J Amer Anim Hosp Assoc 43:337-341

Xipell M, M Bodro, F Marco, RA Losno, C Cardozo, A Soriano (2017). Clinical experience with ceftazidime/avibactam in patients with severe infections, including meningitis and lung abscesses, caused by extensively drug-resistant Pseudomonas aeruginosa. Intl $J$ Antimicrob Agents 49:266-268

Xu Y, H Niu, T Hu, L Zhang, S Su, H He, H Wang, D Zhang (2020). High expression of metallo- $\beta$-lactamase contributed to the resistance to carbapenem in clinical isolates of Pseudomonas aeruginosa from Baotou, China. Infect Drug Resist 13:35-43

Yamaguchi K, R Miyata, R Shigehisa, J Uchiyama, I Takemura-Uchiyama, SI Kato, T Ujihara, Y Sakaguchi, M Daibata, S Matsuzaki (2014). Genome analysis of Pseudomonas aeruginosa bacteriophage KPP23, belonging to the family Siphoviridae. Genome Announc 2; Article e00233-14

Zhu L, J Lin, J Ma, JE Cronan, H Wang (2010). Triclosan resistance of Pseudomonas aeruginosa PAO1 is due to FabV, a triclosan-resistant enoyl-acyl carrier protein reductase. Antimicrob Agents Chemother 54:689-698 